\title{
Erratum: Chronic Subdural Hematoma Spontaneous Resolution
}

\section{Errata: Resolução espontânea de hematoma subdural crônico}

\author{
Nícollas Nunes Rabelo ${ }^{1}$ Vitor Hugo Honorato Pereira ${ }^{1}$ George Santos dos Passos ${ }^{1}$ \\ Luciano José Silveira Filho ${ }^{1}$ André Luiz Cicilini ${ }^{2}$ Neiffer Nunes Rabelo ${ }^{3}$ \\ Luiz Antônio Araujo Dias Junior ${ }^{1}$ Carlos Umberto Pereira ${ }^{4}$ Luiz Antônio Araujo Dias ${ }^{5}$ \\ ${ }^{1}$ Department of Neurosurgery, Hospital Santa Casa, Ribeirão Preto, \\ SP, Brazil \\ 2 Medical Student, Universidade de Ribeirão Preto - Unaerp, Ribeirão \\ Address for correspondence Nicollas Nunes Rabelo, MD, Santa Casa \\ de Ribeirão Preto, Ribeirão Preto, São Paulo, Brazil \\ Preto, SP, Brazil \\ ${ }^{3}$ Medical Student, Faculdade Atenas, Paracatu, MG, Brazil \\ ${ }^{4}$ Department of Neurosurgery, Fundação Beneficiente Hospital de \\ Cirurgia, Aracaju, Sergipe, Brazil \\ ${ }^{5}$ Neurosurgeon and Professor, Department of Neurosurgery, Hospital \\ Santa Casa, Ribeirão Preto, SP, Brazil \\ Arq Bras Neurocir 2017;36:142.
}

\section{ERRATUM}

Rio de Janeiro, May 23, 2017

Dear readers,

In the article Chronic Subdural Hematoma Spontaneous Resolution (doi: 10.1055/s-00371603512), ${ }^{1}$ published online in Arq Bras Neurocir in May 2017, where it reads:

Nícollas Nunes Rabelo ${ }^{1}$ Vitor Hugo Honorato Pereira ${ }^{1}$ Luciano José Silveira Filho ${ }^{1}$ André Luiz Cicilini $^{2}$ Neiffer Nunes Rabelo ${ }^{3}$ Luiz Antônio Araujo Dias Junior ${ }^{1}$ Carlos Umberto Pereira ${ }^{4}$ Luiz Antônio Araujo Dias ${ }^{5}$

It should read:

Nícollas Nunes Rabelo ${ }^{1}$ Vitor Hugo Honorato Pereira ${ }^{1}$ George Santos dos Passos ${ }^{1}$ Luciano José Silveira Filho ${ }^{1}$ André Luiz Cicilini ${ }^{2}$ Neiffer Nunes Rabelo ${ }^{3}$ Luiz Antônio Araujo Dias Junior ${ }^{1}$ Carlos Umberto Pereira ${ }^{4}$ Luiz Antônio Araujo Dias ${ }^{5}$

Prezados leitores,

No artigo Chronic Subdural Hematoma Spontaneous Resolution (doi: 10.1055/s-00371603512), ${ }^{1}$ publicado online em Arq Bras Neurocir em maio de 2017, onde se lê:

Nícollas Nunes Rabelo ${ }^{1}$ Vitor Hugo Honorato Pereira ${ }^{1}$ Luciano José Silveira Filho ${ }^{1}$ André Luiz Cicilini $^{2}$ Neiffer Nunes Rabelo ${ }^{3}$ Luiz Antônio Araujo Dias Junior ${ }^{1}$ Carlos Umberto Pereira ${ }^{4}$ Luiz Antônio Araujo Dias ${ }^{5}$

Lê-se:

Nícollas Nunes Rabelo ${ }^{1}$ Vitor Hugo Honorato Pereira ${ }^{1}$ George Santos dos Passos ${ }^{1}$ Luciano José Silveira Filho ${ }^{1}$ André Luiz Cicilini ${ }^{2}$ Neiffer Nunes Rabelo ${ }^{3}$ Luiz Antônio Araujo Dias Junior ${ }^{1}$ Carlos Umberto Pereira ${ }^{4}$ Luiz Antônio Araujo Dias ${ }^{5}$ 